\title{
Road safety in an aging population: risk factors, assessment, interventions, and future directions
}

\section{Introduction}

With the number of older drivers projected to increase by up to $70 \%$ over the next 20 years, preventing injury resulting from crashes involving older drivers is a significant concern for both policymakers and clinicians. While the total number of fatal crashes per annum has steadily decreased since 2005 in Australia, the rate of fatalities has demonstrated an upward trend since 2010 in drivers aged 65 years and above ( 8.5 per 100,000), such that it is now on par with the fatality rate in drivers aged $17-25$ years (8.0 per 100,000) (Austroads, 2015). Similar statistics are reported for the United States (NHTSA, 2012), implying there is a need for better identification of those older drivers who are unsafe and implementation of strategies that can enhance mobility while maximizing road safety.

The responsibility for ensuring older drivers are safe drivers often falls between the health and road safety sectors, with few countries or jurisdictions providing an overall, integrated approach to assessment, reassessment, and management. Prevalent medical conditions among very old adults (over 85) such as dementia, stroke, and eye disease reduce driving safety and in the case of dementia and macular degeneration will inevitably preclude driving with progression of symptoms.

We argue that there is a need for an evidence-based system of assessment, reassessment, intervention, and license review for older adults. An older driver may present to a psychiatrist, neurologist, optometrist, general practitioner, or other health professional. Reliance solely on health professionals to report crash risk based on clinical judgment may result in patients failing to report important symptoms for fear of license implications. Alternatively, physicians, with little training in relevant evidence-based crash risk assessments, may not recognize deficits associated with unsafe driving. In many countries a system to manage older drivers will need to be multisectorial and multi-disciplinary, involving a range of clinicians (including driving specialists) and government licensing authorities (Carr and O'Neill, 2015).

\section{Clinical judgment of driving capacity without objective assessment}

In most systems, evaluation of fitness to drive (FTD) is largely placed in the hands of physicians. This is consistent across several countries: Canada (Marshall et al., 2012), the United States (American Medical Association, 2014), the United Kingdom, (Hawley and Galbraith, 2008) and Australia (Sims et al., 2012). Given the accessibility of family physicians, and their capacity to screen for a range of medical conditions that potentially impact driving safety, primary care is a logical first point of contact in the process of evaluating FTD. Over the past decade, however, there has been growing recognition that physicians are not confident in making FTD assessments (Lipski, 2002; Jang et al., 2007; Marshall et al., 2012; Sims et al., 2012). Surveys conducted on Canadian physicians from a range of specialties (Jang et al., 2007; Marshall et al., 2012) found that only $33 \%$ felt confident in their ability to make FTD assessments, and $73 \%-88 \%$ felt they would benefit from further education. Similarly, in the Australia surveys of general practitioners (GPs) found that only $41 \%-$ $54 \%$ felt confident in their ability to assess FTD, and $59 \%-74 \%$ felt they would benefit from further education in this area (Lipski, 2002; Sims et al., 2012).

Current recommended practice for FTD assessment in the medical/office-based context includes gathering evidence regarding the patient's cognitive status, presence of fluctuating conditions, visual impairment, musculoskeletal conditions, drugs and alcohol, as well as history of traffic infringements and informant report of driving difficulties (Carr and Ott, 2010; Molnar and Simpson, 2010; Carmody et al., 2012). However, most guidelines for applying this type of clinical data collection are based on expert opinion or consensus statements rather than objective evidence. For example, practice parameters provided by the American Academy of Neurology propose the use of global cognitive measures such as the MiniMental Status Examination (MMSE) and the Clinical Dementia Rating Scale (CDR), and a family/caregiver questionnaire of driving history, 
for identifying patients at risk of unsafe driving (Iverson et al., 2010), with only slightly different protocols suggested by other groups (Canadian Medical Association, 2006; Australian and New Zealand Society for Geriatric Medicine, 2010). Critically, most recommended measures are either not validated against on-road performance, or have poor evidence of association with driving safety risk (Odenheimer et al., 1994; Reger et al., 2004; Crizzle et al., 2012; Joseph et al., 2014). Furthermore, recommendations are not consistent, with some guidelines suggesting a diagnosis of early dementia is sufficient to enforce driving retirement (Iverson et al., 2010; Austroads, 2012), while others suggest this is not sufficient evidence for license cancellation (Australian and New Zealand Society for Geriatric Medicine, 2010).

Few studies have investigated the accuracy or validity of physician assessments of FTD. One study examined the association between physician warnings given to drivers aged over 18 years, and emergency department visits relating to road-traffic incidents in which the patient was a driver $(n=$ 100,075) (Redelmeier et al., 2012). Using a selfmatching cross-over design, Redelmeier et al. found that crash-related injury declined by $45 \%$ in the year following the physician warning compared to the three years preceding the warning. Older drivers, who constituted $51 \%$ of their sample, showed a similar pattern to the rest of the sample with injury rates declining in those aged 60-75 years (3.39 per 1,000 per annum to 2.29 ), and in those aged 75 years and above (2.65 per $1,000 /$ pa to 0.93 per $1,000 / \mathrm{pa}$ ) (Redelmeier et al., 2012). However, the methodology used in this study makes it difficult to determine whether the change was caused by license cancellation (which occurred in 10\%-30\% of cases) or the warning itself, and how the physician warnings were implemented (e.g. driving cessation, self-regulation, or remediation). Importantly, the false positive rate cannot be determined. In fact, Redelmeier and colleagues report that following the physician warning, the patients' emergency department visits for depression increased from 19 per $1,000 /$ pa to 23 per $1,000 /$ pa, and visits to the responsible physician declined by $29 \%$ (Redelmeier et al., 2012) lending some credence to physicians' concerns that FTD assessments have negative impacts on patient-physician relationship and may reduce visitation rates (Lipski, 2002; Jang et al., 2007; Marshall et al., 2012; Sims et al., 2012).

Fox and colleagues (1997) examined the association between physician judgment of FTD in 19 patients with Alzheimer's Dementia (AD) (which included medical exam, MMSE, caregiver report, and vision testing), against an on- road assessment conducted in traffic by an occupational therapist (OT). They found that physician prediction of driving competence was not associated with on-road performance (Fox et al., 1997). Furthermore, $37 \%$ of the AD patients were judged as safe to drive by the on-road assessment, suggesting that $\mathrm{AD}$ diagnosis may not be sufficient to recommend driving cessation.

A Delphi study on physician consensus in identifying at-risk older drivers (Rapoport et al., 2014), had 41 physicians rate 26 patient scenarios, and achieved only $27 \%$ consensus, which improved to $69 \%$ on the fifth iteration. They found the strongest predictors of physician decisions were caregiver concern and abnormal performance on the Clock Drawing Test, and recommended that uncertain cases be referred for on-road assessment (Rapoport et al., 2014). A systematic review of studies on the validity of in-office FTD assessments for a range of chronic diseases (e.g. Parkinson's Disease, AD, Sleep Apnoea, Stroke etc.) concluded that the clinical tests employed in these studies were not consistently related to measures of driving performance (Marino et al., 2013).

Based on current evidence, it is therefore unclear whether physician assessment of FTD provides an accurate prediction of driving safety. Although physician warnings have the potential to influence rates of crash related injury, their specificity, reliability, and accuracy needs to be evaluated in order to minimize negative effects of unnecessary or premature license cancellation. Clear, consistent guidelines and validated in-office screening measures are critical for physicians to not only feel confident in their decision-making for further referral, but to provide an accurate first-tier evaluation of risk in a multi-tiered, multidisciplinary system for assessing FTD (Carmody et al., 2013).

\section{Risk assessment}

Risk assessment for older drivers focusses on cognitive, visual, and motor abilities that are linked to safe driving in large cohort studies (Reger et al., 2004; Mathias and Lucas, 2009; Devos et al., 2011).

\section{Cognitive function}

Common risk assessment tools currently widely used to screen for cognitive impairment fail to discriminate safe from unsafe drivers. For example, a large Canadian study $(n=17,538)$ found that the MMSE did not predict crashes over two years of follow-up (Joseph et al., 2014). Generic tools and dementia screening instruments do not assess the 
specific deficits that cause drivers to become unsafe. A consistent body of research has demonstrated that memory deficits are not typically associated with unsafe driving (Reger et al., 2004; Anstey et al., 2005; Aksan et al., 2015), which may explain why patients with early $\mathrm{AD}$ may continue to drive safely. In contrast, deficits in executive function, visual selective attention, and processing speed predict crashes in older drivers (Ball et al., 2006; Dawson et al., 2010; Anstey et al., 2012). As dementia progresses and multiple domains of cognitive function are affected, driving cessation is inevitable. Some specific types of cognitive deficits in non-AD dementia may also preclude safe driving early in the disease. Cognitive assessment for safe driving focusses on key abilities which are evaluated in most older-driver screening batteries e.g. Wood et al. (2008).

\section{Visual function}

\section{VISUAL TESTS}

Visual acuity (VA) is the most common method employed to assess visual function in clinical practice and by licensing authorities. However, studies using a range of sample sizes and methodologies have found only a weak relationship between VA and crash risk (Hofstetter, 1976; Gresset and Meyer, 1994; Marottoli, 1998; Ivers et al., 1999) or no association (Keeffe et al., 2002; Rubin et al., 2007; Cross et al., 2009). High-contrast static VA is unlikely to represent the normal-driving environment that includes both static and moving objects of different sizes and contrast levels. Tests of contrast and motion sensitivity and visual fields may thus provide better measures of visual performance for driving than VA.

\section{VISUAL AGING AND EYE DISEASE}

Population studies consistently demonstrate an increase in the prevalence of visual impairment for those aged 65 years and above (Klein et al., 1991; Attebo et al., 1996; Rubin et al., 1997). For example, the prevalence of bilateral visual impairment, defined as VA worse than the commonly adopted driving standard of $6 / 12$ (or $20 / 40$ ), increases from $1 \%$ for those between $60-69$ years, to $26 \%$ for those aged 80 years and over (Wang et al., 2000).

An estimated $30 \%$ of the older population have significant cataract in at least one eye (Rochtchina et al., 2003). Many people live with cataracts for extended periods before cataract removal (Owsley et al., 1999); with around a quarter of these continuing to drive, even if their vision fails to meet the visual standard for driver licensing (Monestam, 1999; Pager et al., 2004). This has important ramifications for driving safety, given that older drivers with cataracts have 2.5 times higher crash rates than age-matched controls without cataracts (Owsley et al., 1999).

Glaucoma is one of the leading causes of visual field loss in older adults (Ramrattan et al., 2001) with approximately 60 million people affected by the disease worldwide, with numbers estimated to increase in the aging population (Quigley, 2011). It has been estimated that $20 \%$ of those with glaucoma experience progressive loss of their visual fields even with appropriate treatment (Musch et al., 2009) and approximately half of those with glaucoma are not aware that they have the condition (Weih et al., 2001). Numerous studies suggest that glaucoma is associated with higher at-fault crash risk than those without glaucoma (e.g. McGwin et al. (2015)).

Finally, age-related macular degeneration (AMD) is the leading cause of irreversible moderate to severe visual impairment in older adults worldwide (Weih et al., 2000; Wang et al., 2000). A retrospective study (McGwin et al., 2013) showed that drivers with intermediate AMD, had significantly lower crash rates than drivers with normal vision. The authors suggest that these drivers self-regulate their driving to avoid challenging situations and exercise greater caution on the road, which is reflected in their lower risk for crashes. In contrast, drivers with early and advanced AMD had similar crash rates to the controls. Drivers with early AMD may be less likely to self-regulate their driving, given their better levels of visual function, and thus show similar driving risk profiles to controls. Interpretation of the findings is also difficult for drivers with advanced AMD due to the low numbers of active drivers, as many cease driving in the advanced stages of the condition (Sengupta et al., 2014).

\section{Approaches to prevention}

There is increasing evidence that some interventions, or combinations of interventions, improve the driving safety and mobility of older adults. Adaptive cognitive training programs (i.e. those that systematically increase difficulty level during training), have transferred to both maintained driving mobility and safety. For example, Speed of Processing Training, which focuses on visuallybased processing speed and divided and selective attention, has repeatedly been shown to reduce driving cessation risk across three years and maintain driving mobility across five years as compared to both a no-contact and social-contact control groups (Edwards et al., 2009a; 2009b; Ross et al., 2015). Importantly, this training has also 
demonstrated transfer to driving safety. Ball and colleagues examined the impact of ten hours of three separate cognitive training interventions (Speed of Processing, Reasoning, and Memory) compared to a no-contact control group on crashes across six years (Ball et al., 2010). Results revealed that the Speed of Processing and Reasoning training interventions resulted in over a $40 \%$ reduction in atfault state-reported crashes. When training focused on memory performance, which is not typically predictive of driving outcomes, there was no impact on driving safety.

In addition to improving cognitive function, there is growing evidence that improving visual function related to eye disease has important benefits for road safety. A growing body of work suggests that cataract removal improves driving safety (Subzwari et al., 2008). In one study, cataract surgery resulted in a $50 \%$ reduction in crash rates, compared to those who did not have surgery (Owsley et al., 2002) and an Australian study found that cataract surgery resulted in a $12.7 \%$ reduction in crashes' corresponding to $\$ 4.3$ million in savings (Meuleners et al., 2012). Relatedly, Mennemeyer and colleagues (2013) extended this work using a US data simulation model and found that earlier removal of cataracts, versus current treatment, reduced crashes by $21 \%$, thus illustrating the important, and often under-investigated, impact of the timing of interventions.

Interestingly, educational interventions are one of the most commonly used forms of rehabilitation; however, current evidence suggests that educational interventions alone have a minimal impact on driving safety. Owsley and colleagues found that visually impaired participants reported avoiding more challenging driving situations after an educational intervention (Owsley et al., 2003); however, the intervention had no impact on driving safety (as measured by crashes) two years later (Owsley et al., 2004). Similarly, Bédard and colleagues (2004), examined a widely-used educational intervention and found that the training did not result in significant improvements during an on-road evaluation. There is some evidence that educational programs combined with other components, such as on-road training (Marottoli et al., 2007b; Bédard et al., 2008) may improve onroad driving.

Finally, the improvement of physical abilities related to driving may also be an avenue for improved function. Marottoli and colleagues (2007a) found that a graduated physical therapistguided exercise program translated to improvement of on-road driving performance. Although not an exhaustive review of driving safety interventions, the studies discussed here provide support that there are an increasing number of potential domains, and combinations of domains, that may be targeted to enhance driving safety. Further discussions of driving safety and mobility should include not only assessment, but importantly, potential rehabilitation through evidence-based interventions.

\section{Other older road users - pedestrians and cyclists}

Discussion of road safety in aging often omits walking and cycling which are cost-effective methods of maintaining mobility and increasing physical activity for older adults. Numerous studies report that older adults have the highest risk of mortality of all pedestrians when involved in vehicle collisions (e.g. Koepsell et al., 2002). While this is partly due to older adults' increased frailty, simulation studies indicate that aging affects gap selection, in particular, the ability to weigh-up estimated vehicle time of arrival and one's own walking speed when selecting a gap in the traffic to cross the road (Oxley et al., 2005; Lobjois and Cavallo, 2009; Holland and Hill, 2010; Dommès and Cavallo, 2011). Mobility-impaired older adults show additional difficulty in judging safe crossing gaps to compensate for their rate of walking (Holland and Hill, 2010). Age-related cognitive changes such as decline in executive functions, including decision making, and attentional control, are speculated to contribute to unsafe crossing behavior in older adults (Oxley et al., 2005; Lobjois and Cavallo, 2009; Dommès and Cavallo, 2011). The effects of neurodegenerative diseases such as $\mathrm{AD}$ and Parkinson's disease further increase risk for older pedestrians (Gorrie et al., 2008). Studies suggest that signs of neuropathology (e.g. brain accumulation of neurofibrillary tangles) is associated with risk of older pedestrian crash fatalities (Gorrie et al., 2006), particularly in areas with low complexity traffic (Gorrie et al., 2008).

Cycling in older adults has received very little research interest. However, a Swedish study of older bicyclists $(n=456)$ treated for injury over a ten year period found that the most frequent cause of injury was due to falls when mounting or dismounting the cycle $(20 \%)$ or due to irregular road surfaces $(13 \%)$, with only $6 \%$ due to vehicle collisions (Scheiman et al., 2010). Interestingly, poor balance and falls risk are also associated with reduced executive function in older adults (e.g. Muir et al., 2012), although no study has yet examined cognitive factors in cycling safety. Along with road safety education, cognitive remediation targeting executive functions may help improve 
older adults' safety when cycling or walking within their neighborhoods.

The design of crossings and intersections can also contribute to the elevated risk experienced by older pedestrians (Koepsell et al., 2002). Koepsell and colleagues reported that unless marked crossings are accompanied by traffic signals or signage, they pose much greater risk to older pedestrians relative to un-marked crossings. As such, urban designers may need to take into account how aging affects the ability to safely negotiate traffic, and introduce greater traffic control, separation of pedestrians and vehicles, and pedestrian visibility (Koepsell et al., 2002; Retting et al., 2003; Michael et al., 2006).

Thus, understanding how aging and ageassociated disease impacts pedestrian safety is important for effective promotion of health behaviors, encouraging mobility and independence in aging, and designing age-friendly neighborhoods.

\section{Future directions}

The range of factors that may impact on the capacity to drive safely spans several disciplines. Future strategies for road safety among older adults need to encompass all types of road users, as neurological and sensory deficits impact on the use of public transport, pedestrian safety, and cycling as well as the capacity to access community transport. In most countries there is currently a shortage of driver-trained OTs, and other medical specialists who may be involved in assessing FTD. The ideal scenario is that evidencebased screening and general assessment methods become available which are inexpensive, culturally appropriate, and can be conducted by health care providers with different discipline backgrounds and in different settings. This will allow specialist driving assessment services to focus on complex cases. Evidence-based screening needs to be part of a framework of older driver safety that includes clear referral pathways to interventions, full assessment, and support for driving cessation and alternative transport. Interventions to improve older driver skill and safety are being developed and future investment in research and implementation of these will enhance the overall mobility and quality of life in our aging population.

\section{Conflict of interest}

NT Lautenschlager is the Editor-in-Chief of International Psychogeriatrics and therefore this editorial was assigned to a Deputy Editor of
International Psychogeriatrics for the editorial process.

KAARIN J. ANSTEY, ${ }^{1}$

RANMAlEe ERAMUdugolla, ${ }^{1}$ Lesley A. Ross, ${ }^{2}$

NiCOLA T. LAUTENSCHLAGER ${ }^{3,4}$ AND

JOANNE WOOD ${ }^{5}$

${ }^{1}$ Centre for Research on Ageing, Health and Wellbeing, The Australian National University, Canberra, Australia

${ }^{2}$ Department of Human Development and Family

Studies, The Pennsylvania State University, State

College, USA

${ }^{3}$ Academic Unit for Psychiatry of Old Age, Department of Psychiatry, The University of Melbourne,

Melbourne, Australia

${ }^{4}$ NorthWestern Mental Health, Melbourne Health,

Royal Melbourne Hospital, Melbourne, Australia

${ }^{5}$ School of Optometry and Vision Science and Institute of Health and Biomedical Innovation Queensland

University of Technology, Australia

Email: Kaarin.anstey@anu.edu.au

\section{References}

Aksan, N., Anderson, S. W., Dawson, J., Uc, E. and Rizzo, M. (2015). Cognitive functioning differentially predicts different dimensions of older drivers' on-road safety. Accident Analysis \& Prevention, 75, 236-244.

American Medical Association (2014). Physician's Guide to Assessing and Counselling Older Drivers, 2003. Chicago, IL: American Medical Association.

Anstey, K. J., Horswill, M. S., Wood, J. M. and Hatherly, C. (2012). The role of cognitive and visual abilities as predictors in the multifactorial model of driving safety. Accident; Analysis and Prevention, 45, 766-774.

Anstey, K. J., Wood, J., Lord, S. and Walker, J. G. (2005). Cognitive, sensory and physical factors enabling driving safety in older adults. Clinical Psychology Review, 25, 45-65.

Attebo, K., Mitchell, P. and Smith, W. (1996). Visual acuity and the causes of visual loss in Australia: the Blue Mountains Eye Study. Ophthalmology, 103, 357-364.

Australian and New Zealand Society for Geriatric Medicine (2010). Position statement number 11: driving and dementia. Australasian fournal on Ageing, 29, 137-141.

Austroads (2012). Assessing Fitness to Drive for Commercial and Private Vehicle Drivers: Medical Standards for Licensing and Clinical Management Guidelines. Sydney: Austroads.

Austroads (2015). Review of the National Road Safety Strategy. Research Report AP-R477-15. Sydney: Austroads.

Ball, K. K. et al. (2006). Can high-risk older drivers be identified through performance-based measures in a department of motor vehicles setting? Fournal of the American Geriatrics Society, 54, 77-84.

Ball, K., Edwards, J. D., Ross, L. A. and McGwin, G., Jr. (2010). Cognitive training decreases motor vehicle collision involvement of older drivers. Fournal of the American Geriatrics Society, 58, 2107-2113. 
Bédard, M. et al. (2008). The combination of two training approaches to improve older adults' driving safety. Traffic Injury Prevention, 9, 70-76.

Bédard, M., Isherwood, I., Moore, E., Gibbons, C. and Lindstrom, W. (2004). Evaluation of a re-training program for older drivers. Canadian Fournal of Public Health, 95, 295-298.

Canadian Medical Association (2006). Determining medical fitness to operate motor vehicles. CMA Driver's Guide, 7th edn. Ottawa: CMA.

Carmody, J., Traynor, V. and Iverson, D. (2012). Dementia and driving: an approach for general practice. Australian Family Physician, 41, 230.

Carmody, J., Traynor, V., Iverson, D. and Marchetti, E. (2013). Driving, dementia and Australian physicians: primum non nocere? Internal Medicine fournal, 43, 625-630.

Carr, D. B. and O'Neill, D. (2015). Mobility and safety issues in drivers with dementia. International Psychogeriatrics/IPA, 27, 1613-1622.

Carr, D. B. and Ott, B. R. (2010). The older adult driver with cognitive impairment: "It's a very frustrating life". fAMA, 303, 1632-1641.

Crizzle, A. M., Classen, S., Bédard, M., Lanford, D. and Winter, S. (2012). MMSE as a predictor of on-road driving performance in community dwelling older drivers. Accident Analysis E Prevention, 49, 287-292.

Cross, J. M. et al. (2009). Visual and medical risk factors for motor vehicle collision involvement among older drivers. British fournal of Ophthalmology, 93, 400-404.

Dawson, J. D., Uc, E. Y., Anderson, S. W., Johnson, A. M. and Rizzo, M. (2010). Neuropsychological predictors of driving errors in older adults. Fournal of the American Geriatrics Society, 58, 1090-1096.

Devos, H., Akinwuntan, A. E., Nieuwboer, A., Truijen, S., Tant, M. and De Weerdt, W. (2011). Screening for fitness to drive after stroke a systematic review and meta-analysis. Neurology, 76, 747-756.

Dommès, A. and Cavallo, V. (2011). The role of perceptual, cognitive, and motor abilities in street-crossing decisions of young and older pedestrians. Ophthalmic $\mathcal{E}$ Physiological Optics, 31, 292-301.

Edwards, J. D. et al. (2009b). The longitudinal impact of cognitive speed of processing training on driving mobility. The Gerontologist, 49, 485-494.

Edwards, J. D., Delahunt, P. B. and Mahncke, H. W. (2009a). Cognitive speed of processing training delays driving cessation. Fournals of Gerontology. Series A, Biological Sciences and Medical Sciences, 64, 1262-1267.

Fox, G. K., Bowden, S. C., Bashford, G. M. and Smith, D. S. (1997). Alzheimer's disease and driving: prediction and assessment of driving performance. Fournal of the American Geriatrics Society, 45, 949-953.

Gorrie, C. A., Brown, J. and Waite, P. M. (2008). Crash characteristics of older pedestrian fatalities: dementia pathology may be related to 'at risk' traffic situations. Accident Analysis \& Prevention, 40, 912-919.

Gorrie, C. A., Rodriguez, M., Sachdev, P., Duflou, J. and Waite, P. M. (2006). Increased neurofibrillary tangles in the brains of older pedestrians killed in traffic accidents. Dementia and Geriatric Cognitive Disorders, 22, 20-26.
Gresset, J. and Meyer, F. (1994). Risk of accidents among elderly car drivers with visual acuity equal to $6 / 12$ or $6 / 15$ and lack of binocular vision. Opthalmic $\mathcal{E}$ Physiological Optics, 14, 33-37.

Hawley, C. A. and Galbraith, N. (2008). Medical education on fitness to drive: a survey of all UK medical schools. Postgraduate Medical fournal, 84, 635-638.

Hofstetter, H. W. (1976). Visual acuity and highway accidents. Fournal of the American Optometric Association, 47, 887-893.

Holland, C. and Hill, R. (2010). Gender differences in factors predicting unsafe crossing decisions in adult pedestrians across the lifespan: a simulation study. Accident Analysis \& Prevention, 42, 1097-1106.

Ivers, R. Q., Mitchell, P. and Cumming, R. G. (1999). Sensory impairment and driving: the Blue Mountains Eye Study. American Fournal of Public Health, 89, 85-87.

Iverson, D., Gronseth, G., Reger, M., Classen, S., Dubinsky, R. and Rizzo, M. (2010). Practice parameter update: evaluation and management of driving risk in dementia report of the quality standards subcommittee of the American academy of neurology. Neurology, 74, 1316-1324.

Jang, R. W. et al. (2007). Family physicians' attitudes and practices regarding assessments of medical fitness to drive in older persons. Fournal of General Internal Medicine, 22, 531-543.

Joseph, P. G. et al. (2014). The Mini-Mental State Examination, clinical factors, and motor vehicle crash risk. Fournal of the American Geriatrics Society, 62, 1419-1426.

Keeffe, J. E., Jin, C. F., Weih, L. M., McCarty, C. A. and Taylor, H. R. (2002). Vision impairment and older drivers: who's driving? British fournal of Ophthalmology, 86, $1118-1121$.

Klein, R., Klein, B. E., Linton, K. L. and De Mets, D. L. (1991). The Beaver Dam Eye Study: visual acuity. Ophthalmology, 98, 1310-1315.

Koepsell, T. et al. (2002). Crosswalk markings and the risk of pedestrian-motor vehicle collisions in older pedestrians. fAMA, 288, 2136-2143.

Lipski, P. S. (2002). A survey of general practitioners' attitudes to older drivers on the New South Wales Central Coast. Australasian fournal on Ageing, 21, 98-100.

Lobjois, R. and Cavallo, V. (2009). The effects of aging on street-crossing behavior: from estimation to actual crossing. Accident Analysis \& Prevention, 41, 259-267.

Marino, M. et al. (2013). Interventions to evaluate fitness to drive among people with chronic conditions: systematic review of literature. Accident Analysis E Prevention, 50, 377-396.

Marottoli, R. A. et al. (2007a). A randomized trial of a physical conditioning program to enhance the driving performance of older persons. Fournal of General Internal Medicine, 22, 590-597.

Marottoli, R. A. et al. (2007b). A randomized trial of an education program to enhance older driver performance. Fournals of Gerontology Series A: Biological Sciences and Medical Sciences, 62A, 1113-1119.

Marshall, S., Demmings, E. M., Woolnough, A., Salim, D. and Man-Son-Hing, M. (2012). Determining fitness 
to drive in older persons: a survey of medical and surgical specialists. Canadian Geriatrics fournal, 15, 101.

Mathias, J. and Lucas, L. (2009). Cognitive predictors of unsafe driving in older drivers: a meta-analysis. International Psychogeriatrics, 21, 637-653.

McGwin, G., Jr. et al. (2013). Examining the association between age-related macular degeneration and motor vehicle collision involvement: a retrospective cohort study. British fournal of Ophthalmology, 97, 1173-1176.

McGwin, G., Huisingh, C., Jain, S. G., Girkin, C. A. and Owsley, C. (2015). Binocular visual field impairment in glaucoma and at-fault motor vehicle collisions. Fournal of Glaucoma, 24, 138-143.

Mennemeyer, S. T., Owsley, C. and McGwin, G. (2013). Reducing older driver motor vehicle collisions via earlier cataract surgery. Accident Analysis $\mathcal{E}$ Prevention, 61, 203-211.

Meuleners, L. B., Hendrie, D., Lee, A. H., Ng, J. Q. and Morlet, N. (2012). The effectiveness of cataract surgery in reducing motor vehicle crashes: a whole population study using linked data. Ophthalmic Epidemiology, 19, 23-28.

Michael, Y. L., Green, M. K. and Farquhar, S. A. (2006). Neighborhood design and active aging. Health \& Place, 12, 734-740.

Molnar, F. J. and Simpson, C. S. (2010). Approach to assessing fitness to drive in patients with cardiac and cognitive conditions. Canadian Family Physician, 56, 1123-1129.

Monestam, E. (1999). Impact of cataract surgery. A population based approach. Acta Ophthalmologica Scandinavica, 77, 729

Muir, S. W., Gopaul, K. and Odasso, M. M. M. (2012). The role of cognitive impairment in fall risk among older adults: a systematic review and meta-analysis. Age and Ageing, 41, 299-308.

Musch, D. C., Gillespie, B. W., Lichter, P. R., Niziol, L. M. and Janz, N. K. (2009). Visual field progression in the collaborative initial glaucoma treatment study the impact of treatment and other baseline factors. Ophthalmology, 116, 200-207.

NHTSA (2012). Traffic Safety Facts 2012: Older Population. (pp. http://www-nrd.nhtsa.dot.gov/Pubs/812005.pdf). Washington (DC): National Highway Traffic Safety Administration, Department of Transportation.

Odenheimer, G. L., Beaudet, M., Jette, A. M., Albert, M. S., Grande, L. and Minaker, K. L. (1994). Performance-based driving evaluation of the elderly driver: safety, reliability, and validity. Fournal of Gerontology, 49, M153-M159.

Owsley, C., McGwin, G., Phillips, J. M., McNeal, S. F. and Stalvey, B. T. (2004). Impact of an education program on the safety of high-risk, visually impaired, older drivers. American fournal of Preventive Medicine, 26, 222-229.

Owsley, C., McGwin, G., Jr., Sloane, M., Wells, J., Stalvey, B. T. and Gauthreaux, S. (2002). Impact of cataract surgery on motor vehicle crash involvement by older adults. $\mathcal{F A M A}, 288,841-849$.

Owsley, C., Stalvey, B. T. and Phillips, J. M. (2003). The efficacy of an educational intervention in promoting self-regulation among high-risk older drivers. Accident Analysis and Prevention, 35, 393-400.
Owsley, C., Stalvey, B., Wells, J. and Sloane, M. E. (1999). Older drivers and cataract: driving habits and crash risk. Fournal of Gerontology: Medical Sciences, 54A, M203-M211.

Oxley, J. A., Ihsen, E., Fildes, B. N., Charlton, J. L. and Day, R. H. (2005). Crossing roads safely: an experimental study of age differences in gap selection by pedestrians. Accident Analysis \& Prevention, 37, 962-971.

Pager, C. K., McCluskey, P. J. and Retsas, C. (2004). Cataract surgery in Australia: a profile of patient-centred outcomes. Clinical and Experimental Ophthalmology, 32, 388-392.

Quigley, H. A. (2011). Glaucoma. Lancet, 377, 1367-1377.

Ramrattan, R. S. et al. (2001). Prevalence and causes of visual field loss in the elderly and associations with impairment in daily functioning: the Rotterdam Study. Archives of Ophthalmology, 119, 1788-1794.

Rapoport, M. J. et al. (2014). Developing physician consensus on the reporting of patients with mild cognitive impairment and mild dementia to transportation authorities in a region with mandatory reporting legislation. The American fournal of Geriatric Psychiatry, 22, 1530-1543.

Redelmeier, D. A., Yarnell, C. J., Thiruchelvam, D. and Tibshirani, R. J. (2012). Physicians' warnings for unfit drivers and the risk of trauma from road crashes. New England Fournal of Medicine, 367, 1228-1236.

Reger, M. A., Welsh, R. K., Watson, G., Cholerton, B., Baker, L. D. and Craft, S. (2004). The relationship between neuropsychological functioning and driving ability in dementia: a meta-analysis. Neuropsychology, 18, 85.

Retting, R. A., Ferguson, , S. A. Ferguson and McCartt, A. T. (2003). A review of evidence-based traffic engineering measures designed to reduce pedestrian-motor vehicle crashes. American Fournal of Public Health, 93, 1456-1463.

Rochtchina, E., Mukesh, B. N., Wang, J. J., McCarty, C. A., Taylor, H. R. and Mitchell, P. (2003). Projected prevalence of age-related cataract and cataract surgery in Australia for the years 2001 and 2021: pooled data from two population-based surveys. Clinical and Experimental Ophthalmology, 31, 233-236.

Ross, L. A., Edwards, J. D., O'Connor, M. L., Ball, K. K., Wadley, V. G. and Vance, D. E. (2016). The transfer of cognitive speed of processing training to older adults' driving mobility across 5 years. Fournals of Gerontology Series B: Psychological Sciences and Social Sciences, 71(1), 87-97. doi: 10.1093/geronb/gbv022.

Rubin, G. S. et al. (1997). A comprehensive assessment of visual impairment in a population of older Americans. The SEE Study. Salisbury Eye Evaluation Project. Investigative Ophthalmology and Visual Science, 38, 557-568.

Rubin, G. S., Ng, E. S., Bandeen-Roche, K., Keyl, P. M., Freeman, E. E. and West, S. K. (2007). A prospective, population-based study of the role of visual impairment in motor vehicle crashes among older drivers: the SEE study. Investigative Ophthalmology \& Visual Science, 48, 1483-1491.

Scheiman, S., Moghaddas, H. S., Björnstig, U., Bylund, P.-O. and Saveman, B.-I. (2010). Bicycle injury events among older adults in Northern Sweden: a 10-year 
population based study. Accident Analysis \& Prevention, 42, 758-763.

Sengupta, S., van Landingham, S. W., Solomon, S. D., Do, D. V., Friedman, D. S. and Ramulu, P. Y.

(2014). Driving habits in older patients with central vision loss. Ophthalmology, 121, 727-732.

Sims, J., Rouse-Watson, S., Schattner, P., Beveridge, A. and Jones, K. (2012). To drive or not to drive: assessment dilemmas for GPs. International foumal of Family Medicine. doi: $10.1155 / 2012 / 417512$.

Marottoli, R. A. et al. (1998). Development of a test battery to identify older drivers at risk for self-reported adverse driving events. Fournal of the American Geriatrics Society, 46, 562-568.

Subzwari, S., Desapriya, E., Scime, G., Babul, S., Jivani, K. and Pike, I. (2008). Effectiveness of cataract surgery in reducing driving-related difficulties: a systematic review and meta-analysis. Injury Prevention, 14, 324-328.
Wang, J. J., Foran, S. and Mitchell, P. (2000). Age-specific prevalence and causes of bilateral and unilateral visual impairment in older Australians: the blue mountains eye study. Clinical and Experimental Ophthalmology, 28, 268-273.

Weih, L. M., Nanjan, M., McCarty, C. A. and Taylor, H. R. (2001). Prevalence and predictors of open-angle glaucoma: results from the visual impairment project. Ophthalmology, 108, 1966-1972.

Weih, L. M., VanNewkirk, M. R., McCarty, C. A. and Taylor, H. R. (2000). Age-specific causes of bilateral visual impairment. Archives of Ophthalmology, 118, 264-269.

Wood, J. M., Anstey, K. J., Kerr, G. K., Lacherez, P. F. and Lord, S. (2008). A multidomain approach for predicting older driver safety under in-traffic road conditions. Fournal of the American Geriatrics Society, 56, 986-993. 